\title{
Consistent Condom Use in HIV/AIDS Patients Receiving Antiretroviral Therapy in Northwestern Ethiopia: Implication to Reduce Transmission and Multiple Infections - A Critical Review of Article
}

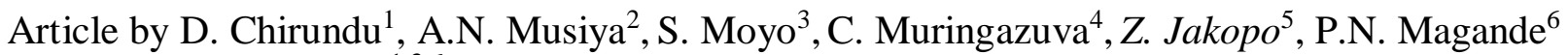 \\ 1,3,6 Kadoma City Health Department, Zimbabwe \\ ${ }^{2}$ Department of Community Medicine, University of Zimbabwe, Zimbabwe \\ ${ }^{4}$ Zimbabwe Defence Forces $H Q$, Zimbabwe \\ ${ }^{5}$ Kadoma General Hospital, Zimbabwe \\ ${ }^{6}$ Kadoma City Health Department, Zimbabwe \\ E-mail:dchirundu@me.com ${ }^{1}$,drnyashaadrianmusiya@gmail.com ${ }^{2}$,skhaemoyo@gmail.com ${ }^{3}$, \\ muringac@gmail.com ${ }^{4}$,jzorodzai@gmail.com ${ }^{5}$,pmagande@gmail.com ${ }^{6}$
}

Source: Zewdneh Shewamene et. al. 2015, "Consistent condom use in HIV/AIDS patients receiving antiretroviral therapy in northwestern Ethiopia: implication to reduce transmission and multiple infections" HIV/AIDS- Research and Palliative Care, 2015:7 119-124 downloaded 10 August 2015 http://www.dovepress.com/articles.php?article_id=21307

\begin{abstract}
Introduction: This is a critical review of an article by Zewdneh Shewamene published in the July 2015. The central theme is that consistent condom use among HIV patients is important in preventing transmission of HIV.

Article summary: The premise of the authors is that consistent condom use among HIV infected is critical for interrupting transmission of HIV and acquisition of resistant strains of HIV. Among the 317 respondents, condoms awareness was 96.2\%. Independent factors for consistent use were, male sex (AOR=6.87; $p=0.001)$, urban residency, $(A O R=4.65 ; p=0.001)$, a higher education attainment $(A O R=8.98 ; p=0.001)$, and prolonged duration on $A R T(A O R=3.91 ; p=0.001)$. The conclusion was that some HIV infected people were not using condoms.

Article critique: The article maybe considered as objective, and unbiased due to the peer review process. The journal that published it is indexed on PubMed, and, Embase, among others. That the article was published in a journal for those with an interest in HIV and palliative care makes it relevant for HIV programming particularly in Africa where HIV burden is high. The objectives are well spelt, and the methodology is easy to follow. Only verbal consent was obtained from the respondents contrary to the dictates of the Belmont report that requires written informed consent.

Conclusion: The articles contributed to literature and can provide a basis of improving future studies of a similar nature. However, it is suggested that measurement of condom use should be serial and be recoded to a dichotomous variable in analysis.
\end{abstract}

Keyword: Condoms, Ethiopia, HIV.

\section{Introduction}

This is a critical review of an article entitled "Consistent condom use in HIV/AIDS patients receiving antiretroviral therapy in northwestern Ethiopia: implication to reduce transmission and multiple infections" published in the July 2015 issue of the HIV/AIDS- Research and Palliative Care journal. The corresponding author is Zewdneh Shewamene. This review will firstly give an overview of the article. Secondly the article structure will be reviewed on the presentation of the content, the alignment and format of the article. Thirdly 
DOI: $10.21522 /$ TIJPH.2013.06.02.Art012

ISSN: $2520-3134$

the article will be critiqued looking at the authority, accuracy, currency, relevance, objectivity and coverage. Tables will also be looked at before judging the article's accessibility and credibility.

The central theme of the article is that consistent condom use among HIV/AIDS patients is an important method of preventing transmission and acquiring of infections. This includes sexually transmitted infections including HIV, and reinfection with resistant strains of HIV. It is against this background that the frequency and determinant factors of consistent condom use were investigated among attendees of a university Antiretroviral Therapy clinic in Ethiopia. This is generally a well thought and presented article. It is very relevant to Sub-Saharan Africa which accounts for almost $67 \%$ of the global total of new HIV infections (UNAIDS 2017). Under such circumstances correct and consistent condom use is a feasible method of preventing reinfection (Population Action International 2002).

\section{Article summary}

The background of HIV globally and in Ethiopia is proffered. An elaboration is done on availability of free condoms in the country. The premise of the authors is that consistent condom use among HIV infected is critical as it prevents transmission of HIV to uninfected partners, acquisition of resistant strains of HIV, and, impacts on community HIV prevalence.

The study was a hospital based analytical cross-sectional study that had 317 randomly selected respondents. Data were collected using a pretested questionnaire. The dependent variable was consistent use of condom in every sexual encounter in the six months prior to the study. Independent variables included socio-demographic variables and general awareness regarding condoms. Data analysis was done using SPSS. The study was funded and approved by the University of Gondar in Ethiopia. Verbal consent was obtained from participants.

Among the 317 respondents, $49.8 \%$ were males and 50.2\% (159) were females. Condom awareness was 96.2\%. Independent factors for consistent condom use were, male sex (AOR=6.87; $\mathrm{p}=0.001$ ), urban residency, $(\mathrm{AOR}=4.65 ; \mathrm{p}=0.001)$, a higher education attainment $(\mathrm{AOR}=8.98 ; \mathrm{p}=0.001)$, and prolonged duration on $\mathrm{ART}(\mathrm{AOR}=3.91 ; \mathrm{p}=0.001)$.

The authors concluded that some HIV infected people were not using condoms. However, in the discussion the "implication to reduce transmission and multiple infections" as alluded to in the title was not articulated. The article nevertheless, is well presented, has 13 subheadings, ensuring wide coverage of issues. There are citations throughout the article that allows the reader to evaluate findings and thesis against other works.

\section{Article structure}

The article has an abstract. In the main body there is the introduction that covered the literature review, objectives and rationale of the study. Under the methodology, subheadings of study area, study design and sampling, data collection procedures are covered. The dependent and independent variables are well defined under the data collection section. Data analysis is also another subheading within the methodology. Ethics section is also included. Under the results section, there are 3 subheadings covering study population characteristics, general condom awareness and the determinants of consistent condom use which are presented as tables. Recommendations and conclusions form the last part of the main body. There is a section on acknowledgements, author contributions and disclosure. The structure is therefore coherent and allows the articulation of pertinent issues in detail and depth.

\section{Article critique}

\section{Authority}

The article was published by an international peer reviewed open access journal that focuses on research in HIV, "The Journal of HIV/AIDS Research and Palliative care (ISSN 1179-1373)" This may be considered to be an objective, unbiased publication due to the peer review process. It is indexed on PubMed, 
Embase, Directory of Open Access Journal and the OAlster Open Initiative. These are all credible databases. The journal is also a member of the Committee on publication ethics.

The corresponding author was a member of the faculty of pharmacology at University of Gondor in Ethiopia. He holds a master's degree in Pharmacology and is currently study for his $\mathrm{PhD}$ with University of Western Sydney Australia. He has published 27 peer reviewed articles in a variety of journals. The project under review was funded by the University of Gondor; this affords it further credence.

\section{Accuracy}

The article is based on field work that was done from April to May 2013. It is backed up by a reference list that is cited in text and can be easily followed by reader. The peer review process, editorial processing and referencing, all contributed to the accuracy of the study. Under the author contribution it is indicated that "All authors reviewed and approved the final manuscript prior to submission and agree to be accountable for all aspects of the work." This is a further attestation that the authors stand by the accuracy of their work.

\section{Currency}

The article was published in July 2015. However, its date of submission was not reported. Data were collected between April and May 2013. All these factors make the article relatively current.

\section{Relevance}

This article was published in a peer reviewed journal for those with an interest in HIV and palliative care. It was written to inform both researchers and those in HIV programming. It is very relevant for HIV programming particularly in Africa which is burdened with a high prevalence of HIV. Under the circumstances condoms are a recommended intervention (Lurie et. al. 2008). In the developed world (and some parts of Africa) it can be used for programming in men who have sex with men. However, looking at the title and the content, the articles falls short of articulating the "implication to reduce transmission and multiple infections".

\section{Objectivity}

The objectives are clear and well spelt out. The methodology is easy to follow, and the study can be repeated in other settings. Clear methodology and objectives reduces bias and facilitates repeatability. However, the outcome variable consistent condom use was a dichotomous yes/ no. Given that the condom use is a continuous phenomenon, one would argue that this dichotomous measurement of condom use is not a sensitive way to measure condom use. When several response alternatives are provided to a condom frequency question, a researcher can gain reasonable insight into how often one uses condoms (Noar et. al 2006).

The recall period in the study was six months. Whilst a longer recall period may provide a better representation of the sexual behavior, little research has demonstrated the accuracy of recall periods of more than 3 months (Sheeran et. al. 1994 and Schroder et. al. 2003). This may introduce recall bias. It would also have made the article more specific to indicate if the intercourse was vaginal sex or anal sex or it was man having sex with man.

Whilst ethical clearance was obtained from the University, it is not clear if it were ordinary university authorities or a university ethical review board. Only verbal consent was obtained from the respondents contrary to the dictates of the Belmont report, that requires written informed consent (The National Commission for the Protection of Human Subjects of Biomedical and Behavioral Research (1978) and William et. al. 2008) ${ }^{1}$. The respondents were clients seeking care at a hospital. It is a known that there is a difference among those who seek care and those who do not. This may affect programming as people with a shorter duration or not on ART are not likely to use condoms consistently (Alene et. al. 2014). Nevertheless, the article can be deemed objective. The references cited were published between 1997 and 2011. However, citation of later publications would have made it more objective. 
DOI: $10.21522 / \mathrm{TIJPH} .2013 .06 .02 . A r t 012$

ISSN: $2520-3134$

\section{Stability}

The paper is stable since it was published in a peer reviewed journal. Other researchers and program managers can depend on it for guidance.

\section{Analysis of tables}

There were no graphs in the article. However, there are 3 tables that are adequately labelled and abbreviations appearing in them are explained e.g. AOR.

\section{Recent advances related to the topic}

Condom use is documented as one of the measures that can prevent sexual transmission of HIV (UNAIDS 2003; UNFPA 2002 and Cohen et. al 1999) ${ }^{1}$. In 2007, the Global Alliance on Condom use identified social and cultural, legal/policy, economic/financial and structural barriers as barriers to condom use (Drezin et. al 2007 and Daly et. al 2009). According to UNAIDS (2006) condoms if used correctly and consistently, can reduce the risk of sexual transmission by 80-90 percent. So far, condoms are the only product that can effectively protect against HIV and other sexually transmitted infections (Population Action International 2002). With the improved access to ART some infected people now have suppressed viral loads. With the subsequent improvement in health-related quality of life some of the HIV infected people are engaging in sexual risky behavior, and, this calls for accelerated condom promotion in this subpopulation (Stole et. al.2001 and Pedraza et. al. 1999).

Nonetheless, several studies in different geographical locations have reported low condom use among the HIV infected. Moazen et. al, (2017) reported a consistent condom use rate of $16.7 \%$ in a study carried out in Iran. In a study carried out in Zimbabwe (2016), Chirundu reported a 37\% and 52\% consistent condom use among males and females on ART respectively. Perceptions that condoms reduce pleasure; perception that there is no need for condoms in long term unions all militated against consistent condom use. Macharia et. al. (2017) also reported reduced sexual pleasure and non-disclosure of HIV status as barriers to condom use among the HIV infected. Furthermore, Goncalves et. al. in a system review published in 2017 concluded that behavioural interventions alone may not be adequate to promote consistent condom use among the HIV infected.

Nakaie et. al. (2014) in a study done in Cambodia, reported that the patient's formal education, gender, type of partner (regular or casual) or number of sexual partners were not significantly associated with consistent condom use but the "ability to ask a partner to use condom at every sexual intercourse" was a predictor. In an Italian study among the HIV infected, the prevalence of condom use was 44\%. Less income, lower education and having one child were negatively associated with condom use. Alene et. al (2014) in a study done in Ethiopia found out that those who had a higher level of education and have been on ART for longer periods were more likely to report condom use. Lurie et. al. (2008) in a study among the HIV infected patients in South Africa found predictors of consistent condom being regular partners, urban residence, and higher education levels disclosure and younger age.

In Zimbabwe McClellan et. al. (2010). Reported low condom use was among HIV-positive, aboveaverage educated women. Less than half reported ever-use of condoms, being a younger woman, being currently married, living with current husband/partner, religion, longer duration since HIV diagnosis (years), disclosure of HIV status to current husband/partners, and greater number of sexual partners in the past year predicted consistent condom use.

Canto et. al (2015) in a study carried out in the Dominican Republic found no association between alcohol consumption and unprotected use. This is contrary to previous reports (Kalichman S.C. 2000. and Crepaz N. \& Marks G. 2002).

\section{Conclusion}

This was a critical review of Zewdneh Shewamene's article "Consistent condom use in HIV/AIDS patients receiving antiretroviral therapy in northwestern Ethiopia: implication to reduce transmission and 
multiple infections". The content, structure and limitations of the study were analyzed and and suggestions made. The article has contributed to literature and can provide a basis of improving future studies of a similar nature. However, it is suggested that measurement of condom use should be serial and be recoded to a dichotomous variable in analysis. It is also suggested that the recall period be 3 months or less as recommended by other studies. Condoms may be used for vaginal or anal sex and it would give the reader more clarity if same is specified. A study on condom use among HIV infected in the general population not seeking care is also recommended.

\section{Acknowledgements}

We acknowledge the contribution of Prince Matambo and Patrick Makovere for typesetting this manuscript.

\section{References}

[1]. Alene et. al. (2014). Consistent condom use among sexually active HIV-positive women in Amhara region, Ethiopia. Open Access Journal of Contraception 2014:5 85-90.

[2]. Canto et. al. (2015) Substance Use and Condom Use among the HIV Population at Clínica de Familia La Romana, Dominican Republic. JGH Vol. V Issue I.

[3]. Chirundu Daniel (2016). Condom Use among HIV infected clients seeking care at Rimuka Integrated HIV and Tuberculosis Site, Kadoma, Zimbabwe 2015. South American Journal of Public Health Special Edition.

[4]. Cohen D et. al. (1999) Condom Skills, Education and Sexually Transmitted Disease Re-infection. Journal of Sex Research, 28 (1), 139-144.

[5]. Crepaz N. and Marks G. (2002). Towards an understanding of sexual risk behavior in people living with HIV: a review of social, psycho- logical, and medical findings. AIDS, 16(2), 135-49.

[6]. Daly et. al. (2009), Callie Long and Mary Ann Torres. Barriers to condoms - Implementing and documenting advocacy strategies. International Council of AIDS Service Organizations (ICASO).

[7]. Drezin et. al. (2007). Barriers to Condom Access: setting an advocacy agenda. International Council of AIDS Service Organizations (ICASO).

[8]. Haddad et. al. (2018) Factors associated with condom use among men and women living with HIV in Lilongwe, Malawi: a cross sectional study BMJ Sex Reprod Health2018; 44:42-53.

[9]. Kalichman S.C. (2000). HIV transmission risk behaviors of men and women living with HIV-AIDS: prevalence, predictors, and emerging clinical interventions. Clinical Psychology Science and Practice, 7, 32-47.

[10]. Lurie et. al. (2008) Sexual Behavior and Reproductive Health among HIV-Infected Patients in Urban and Rural South Africa; J Acquir Immune Defic Syndr.

[11]. Macharia et al. (2017) Barriers of Condom Use among HIV Positive Women at Thika Level 5 Hospital, Kenya J AIDS Clin Res 2017, 8:8 DOI: 10.4172/2155-6113.1000722.

[12]. McClellan et. al. (2010). Fertility desires and condom use among HIV-positive women at an antiretroviral rollout program in Zimbabwe. Afr J Reprod Health: 27-35.

[13]. Noar et. al. (2006) Condom Use Measurement in 56 Studies of Sexual Behavior: Review and Recommendation: Archives of Sexual Behavior.

[14]. Naomi Nakaie et. al. (2014) Family planning practice and predictors of risk of inconsistent condom use among HIV-positive women on anti-retroviral therapy in Cambodia: BMC Public Health 2014, 14:170

[15]. Population Action International. (2002) Condoms count. Meeting the need in the era of HIV/AIDS. The PAI report card.

[16]. Pedraza MA et. al. (1999) Heterosexual transmission of HIV-1 is associated with high plasma viral load levels and a positive viral isolation in the infected partner. J Acquir. Immune Defic Syndr 21: 120 - 125.

[17]. Schroder, K. E. et. al. (2003). Methodological challenges in research on sexual risk behavior: I. Item content, scaling, and data analytical options. Annals of Behavioural Medicine, 26, 76-103.

[18]. Sheeran, P., \& Abraham, C. (1994). Measurement of condom use in 72 studies of HIV-preventive behaviour: a critical review. Patient Education and Counseling, 24(3), 199-216. DOI: 10.1016/0738-3991(94)90065-5. 
DOI: $10.21522 / \mathrm{TIJPH} .2013 .06 .02 . A r t 012$

ISSN: $2520-3134$

[19]. Stole IG et.al. (2001) Increase in sexually transmitted infections among homosexual men in Amsterdam in relation to HAART. Sex Transm Infect 77: $184-186$.

[20]. The National Commission for the Protection of Human Subjects of Biomedical and Behavioral Research. (1978)

The Belmont report: Ethical Principles and Guidelines for the Protection of Human Subjects of Research. . U.S. Government Printing Office.

[21]. Williams et. al. (2008) The Declaration of Helsinki and Public health. Bulletin of the World Health Organization 2008; 86: 650-651 A Guide to Research Ethics. University of Minnesota Centre for Bioethics

[22]. UNAIDS (2003). Condoms for HIV Prevention: An Analysis of the Scientific Literature. (Discussion Paper). UNAIDS.

[23]. UNAIDS (2006). Report on the global AIDS Epidemic UNAIDS.

[24]. UNAIDS (2017), 2017 UNAIDA Data. UNAIDS.

[25]. UNFPA 2002. Condom Programming for HIV Prevention. HIV Prevention NOW. Programme Briefs. No. 6. New York: UNFPA. 2002.

[26]. Younge (2008). Condom Use at last sex as a proxy for other measures of condom use: Is it Good Enough? Adolescence 2008: (172): 927-931). 